\title{
Multimoded reflective delay lines and their application to resonant delay line rf pulse compression systems
}

\author{
Sami G. Tantawi \\ SLAC, Menlo Park, California 94025, USA \\ (Received 8 December 2003; published 2 March 2004)
}

\begin{abstract}
Pulse compression systems for future linear colliders, such as NLC and JLC, involve hundreds of kilometers of waveguide runs. These waveguides are highly overmoded to reduce the rf losses. In this paper we present a novel idea for utilizing these waveguides several times by using different modes. This idea is suitable for reflective delay lines. All the modes being used have low-loss characteristics. We describe mechanically simple mode transducers that switch the propagation mode from one configuration to another with no observable dispersion. We apply this technique to a resonant delay line pulse compression system. We also present experimental results that verify these theoretical developments.
\end{abstract}

DOI: 10.1103/PhysRevSTAB.7.032001

PACS numbers: $84.40 . \mathrm{Az}, 84.40 . \mathrm{Dc}$

\section{INTRODUCTION}

The rf pulse compression systems for future $X$-band linear colliders [1] contain very long runs of overmoded waveguides. A typical system might contain a few $100 \mathrm{~km}$ of circular waveguide under vacuum. Reducing the length of these waveguides by loading them with disks and irises increases the losses of the system. Loading, also, makes the waveguide dispersive and distorts rf pulse shape. To reduce the lengths of these runs, multimoded rf structures and transmission lines have been suggested [2]. In these multimoded systems, the transmission lines transmit the rf power in several modes utilizing the transmission line several times.

Here we suggest a variation which is suitable for reflective delay lines. These are used in systems such as the resonant delay line pulse compression system, also known as SLED-II [3,4]. The scheme suggested here can reduce the required delay line by a factor of $n$, where $n$ is the number of modes used simultaneously. We present a design for two modes and show experimental data for that scheme. Then we consider the problem of using this technique for a high efficiency high power resonant delay line pulse compression system. We present component designs for an $X$-band system with a compression ratio of 4 and an output pulse width of $400 \mathrm{~ns}$ and present experimental results for such a system.

\section{MULTIMODED REFLECTIVE DELAY LINES}

Consider the delay line shown in Fig. 1. The rf signal is injected from the left into the delay line waveguide in the $\mathrm{TE}_{01}$ mode. We choose the dimensions of the input port such that only this azimuthally symmetric TE mode can propagate. The waveguide is then tapered up to a diameter that supports several $\mathrm{TE}_{0 n}$ modes. The $\mathrm{TE}_{01}$ mode travels all the way to the end of the delay line and then gets reflected. However, the end reflector also converts the reflected mode into the $\mathrm{TE}_{02}$ mode. The $\mathrm{TE}_{02}$ mode travels back to the beginning of this line and, since the input of the line cuts off this mode, it is reflected. If the input taper is designed carefully, the $\mathrm{TE}_{02}$ mode can be completely reflected without scattering into other modes. Then, because of reciprocity, the $\mathrm{TE}_{02}$ wave is converted back to $\mathrm{TE}_{01}$ at the other end of the line. This mode then travels back and exits the line. The total delay in the delay line is twice that seen by a single-moded line. Hence, one can cut the delay line length by a factor of 2 .

This scheme can be repeated for more than two modes. For example one can use $\mathrm{TE}_{01}, \mathrm{TE}_{02}, \mathrm{TE}_{03}$, and $\mathrm{TE}_{04}$ for a factor-of-4 reduction in length. In this case the end taper has to both transform the $\mathrm{TE}_{01}$ mode, on reflection, to the $\mathrm{TE}_{02}$ mode and transform the $\mathrm{TE}_{03}$ mode, on reflection, to the $\mathrm{TE}_{04}$ mode. The input taper has to transmit the $\mathrm{TE}_{01}$, reflect the $\mathrm{TE}_{02}$ into the $\mathrm{TE}_{03}$ mode, and reflect the $\mathrm{TE}_{04}$ mode into itself. The design techniques presented below allow us to produce tapers with these properties. Designs for four-mode systems have been presented by the author [5]. Designs for three-mode systems using adiabatic tapers have been presented by Kazakov [6]. However, these designs are still in their initial stages and have no supporting experimental data.

\section{THE END MODE CONVERTER}

The mode converter at the end of the delay line is shown in Fig. 2. It is basically a step in the circular waveguide. If the big waveguide supports only the $\mathrm{TE}_{01}$ and the $\mathrm{TE}_{02}$ modes among all $\mathrm{TE}_{0 n}$ modes and the small waveguide supports only the $\mathrm{TE}_{01}$ mode, one can choose the diameter of the small guide so that the power transmitted from the large guide to the small guide at the step is independent of which mode is incident. That is, the coupling between the $\mathrm{TE}_{01}$ mode in the large waveguide and the $\mathrm{TE}_{01}$ in the small waveguide is equal in magnitude to the coupling between the $\mathrm{TE}_{02}$ in the large waveguide and the $\mathrm{TE}_{01}$ in the small waveguide. Then the junction can be viewed as a symmetrical, loss-less, and reciprocal three-port device with two similar ports, 


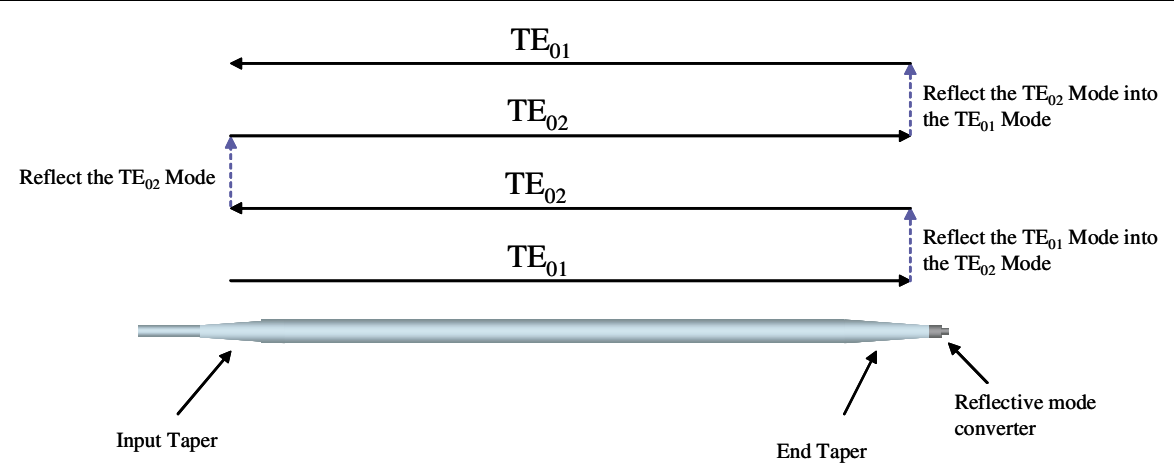

FIG. 1. (Color) Dual-moded delay line.

namely, port 1 (representing the $\mathrm{TE}_{01}$ in the large guide) and port 2 (representing the $\mathrm{TE}_{02}$ in the large guide).

The scattering matrix for such a network is unitary and symmetric. By imposing these two constraints on the scattering matrix $\underline{S}$ of our device and at the same time taking into account the symmetry between port 1 and port 2 , at some reference planes, one can write

$$
\underline{S}=\left(\begin{array}{ccc}
\frac{e^{j \phi}-\cos \theta}{2} & \frac{-e^{j \phi}-\cos \theta}{2} & \frac{\sin \theta}{\sqrt{2}} \\
\frac{-e^{j \phi}-\cos \theta}{2} & \frac{e^{j \phi}-\cos \theta}{2} & \frac{\sin \theta}{\sqrt{2}} \\
\frac{\sin \theta}{\sqrt{2}} & \frac{\sin \theta}{\sqrt{2}} & \cos \theta
\end{array}\right) .
$$

With the proper choice of the reference planes, this expression is quite general for any symmetric three-port network. The scattering matrix properties are determined completely with only two parameters: $\theta$ and $\phi$. The scattered $\mathrm{rf}$ signals $\underline{V}^{-}$are related to the incident $\mathrm{rf}$ signals $\underline{V}^{+}$by

$$
\underline{V}^{-}=\underline{S V^{+}}
$$

where $V_{i}^{ \pm}$represents the incident/reflected rf signal from the $i$ th port. We terminate the third port so that all the scattered power from that port is completely reflected, i.e.,

$$
V_{3}^{+}=V_{3}^{-} e^{j \psi}
$$

The resultant, symmetric, two-port network, then, has the following form:

$$
\underline{S}_{\text {two-port }}=\left(\begin{array}{cc}
\cos \left(\frac{\zeta-\phi}{2}\right) e^{j[(\phi / 2)+(\zeta / 2)+\alpha]} & j \sin \left(\frac{\zeta-\phi}{2}\right) e^{j[(\phi / 2)+(\zeta / 2)+\alpha]} \\
j \sin \left(\frac{\zeta-\phi}{2}\right) e^{j[(\phi / 2)+(\zeta / 2)+\alpha]} & \cos \left(\frac{\zeta-\phi}{2}\right) e^{j[(\phi / 2)+(\zeta / 2)+\alpha]}
\end{array}\right),
$$

where the angle $\zeta$ is given by

$$
e^{j \zeta}=\frac{\cos \theta-e^{j \psi}}{\cos \theta e^{j \psi}-1}
$$

and $\alpha$ is an arbitrary angle added to (4) so that the reference planes can be chosen at will.

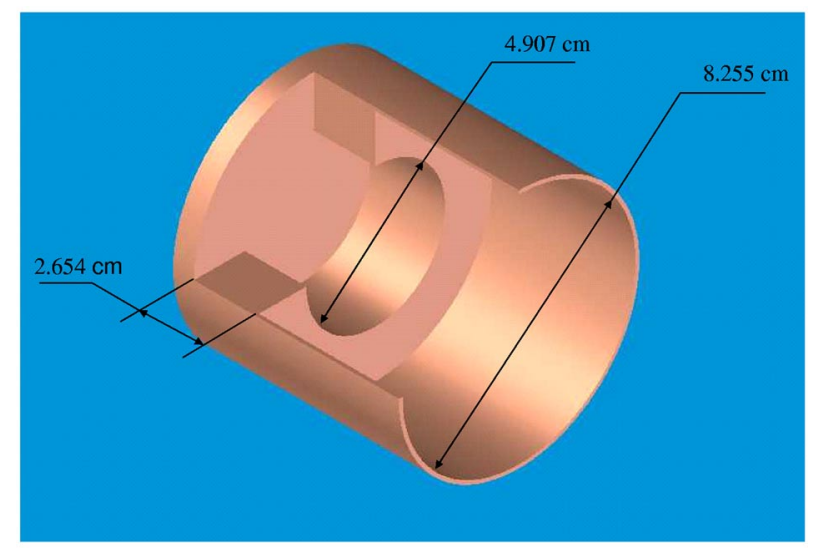

FIG. 2. (Color) $\mathrm{TE}_{01}-\mathrm{TE}_{02}$ reflective mode converter. The dimensions shown are for an operating frequency of $11.424 \mathrm{GHz}$.
From Eq. (5), changing the angle $\psi$ of the third port terminator can set the angle $\zeta$ to any desired value. Hence, from Eq. (4) the coupling between the first and the second ports can be varied from 0 to 1 .

The design procedure for this reflective mode converter can be summarized as follows: one chooses the relative diameters between the small and large waveguides such that the couplings of both the $\mathrm{TE}_{01}$ and the $\mathrm{TE}_{02}$ modes in the large waveguide are equal. Then, according to the above theory, there exists a position for a short circuit termination such that the coupling between the $\mathrm{TE}_{01}$ mode and the $\mathrm{TE}_{02}$ mode in the large waveguide is equal to 1 . Choosing the diameters and then finding the location of the short circuit was done using numerical mode matching codes and verifying with the finite element code, HFSS [7].

The only step left in the design of this end mode converter is a careful taper design that transforms the diameter of the delay line into the diameter of a waveguide that can support only $\mathrm{TE}_{01}$ and $\mathrm{TE}_{02}$ modes. The taper needs to transfer both modes perfectly. We used adiabatic tapers for the initial proof of principle experiment. We then used more sophisticated design techniques 
for the pulse compression design and experiment; see Sec. V.

\section{PROOF OF PRINCIPLE EXPERIMENT}

To prove the concept we used a $12.065 \mathrm{~cm}$ diameter waveguide for the delay line. At our operating frequency of $11.424 \mathrm{GHz}$, this line supports four $\mathrm{TE}_{0 n}$ modes. We used adiabatic tapers for both ends of the line. The design techniques used for these tapers are similar to those used in [8]. With a line length of $35.35 \mathrm{~m}$ the delay through the line is about $75 \mathrm{~ns}$. With a short circuit at the end we get a round-trip delay of about $150 \mathrm{~ns}$, shown in Fig. 3(a). The
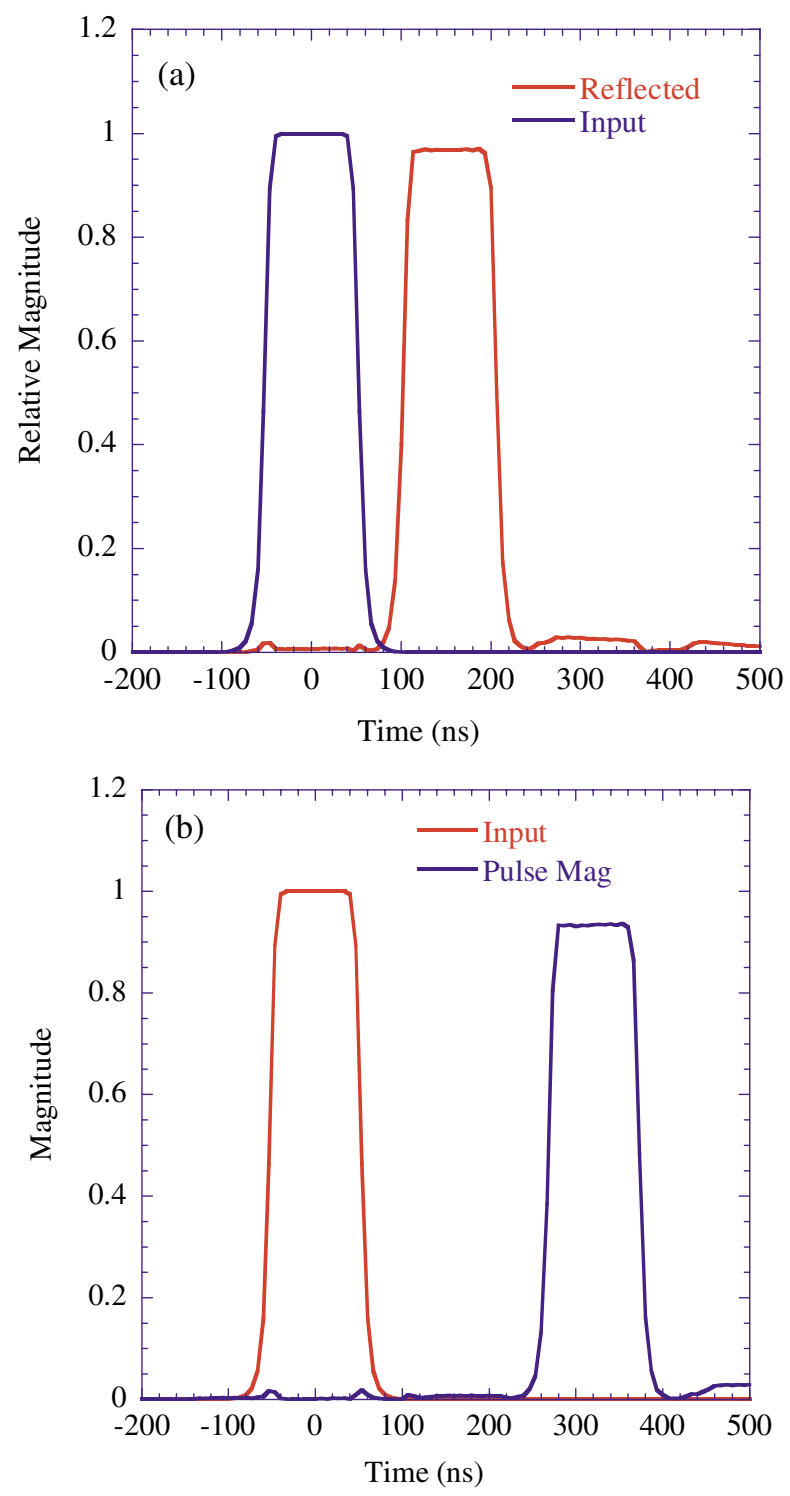

FIG. 3. (Color) (a) Measured delay through $75 \mathrm{ft}$ of WC475 waveguide terminated with a flat plate. The round-trip delay time is $154 \mathrm{~ns}$. (b) Measured delay through $75 \mathrm{ft}$ of WC475 waveguide terminated with the $\mathrm{TE}_{01}-\mathrm{TE}_{02}$ mode converter. The round-trip delay time is $320 \mathrm{~ns}$. The operating frequency is $11.424 \mathrm{GHz}$. measurement techniques are reported in [3] and summarized in Sec. VC. Figure 3(b) shows the delay after placing the mode converter at the end of this line. The delay was doubled at the expense of increased loss. Using a larger diameter waveguide for the delay line can reduce the loss.

\section{DUAL-MODE RESONANT DELAY LINE PULSE COMPRESSION SYSTEM}

\section{A. Resonant delay line design}

At the NLC required pulse length of $400 \mathrm{~ns}$ [1], the dual-moded delay line needs to have an approximate length of $30 \mathrm{~m}$. At this length, using both the $\mathrm{TE}_{01}$ and the $\mathrm{TE}_{02}$ modes in a single delay line forced us to increase the line diameter to reduce the losses. The diameter of choice for this line is $17.082 \mathrm{~cm}$. In a continuous interval of diameters that have acceptable losses this particular choice makes the waveguide delay line operate as far as possible away from any TE or TM modes that have a cutoff frequency near our operating frequency of $11.424 \mathrm{GHz}$. This helps reduce losses due to spurious mode excitation.

At this diameter, there are six possible $\mathrm{TE}_{0 n}$ modes that can propagate. Designing adiabatic tapers at this level of overmoding results in tapers that are approximately $1 \mathrm{~m}$ long. To make the manufacturing of these tapers manageable, we introduced in the next section a new design technique for these tapers. Also the delay line needs to be adjusted in length to bring it into resonance [3]. This means that the mode converter at the end needs to be movable. We had two choices, either to combine the end taper with the mode converter and move the assembly as a whole or to split the reflective mode converter at the plane separating its large waveguide from its small waveguide and only move the latter [9]. We chose the latter solution. This is shown in Fig. 4. We chose a gap between the movable cup and the waveguide wall of about $0.76 \mathrm{~mm}$. This is possible because $\mathrm{TE}_{0 n}$ modes do not have axial currents.

We used the same design technique for the end taper and for the input taper. Then, we added an iris at the small diameter of the input taper where the $\mathrm{TE}_{01}$ mode is the only propagating mode among all $\mathrm{TE}_{0 n}$ modes. This iris has a reflection coefficient, for the $\mathrm{TE}_{01}$ mode, of 0.607 , the optimum iris for a compression ratio of 4 [3].

\section{B. Input and end-taper designs}

We note that the $\mathrm{TE}_{0 n}$ modes have neither axial currents nor normal electric fields on the waveguide walls. This allows the design of these tapers in steps using abrupt transitions. We gave up completely the idea of adiabaticity. We divided the tapers into several segments and used a mode matching code to simulate their response. For a detailed description of mode matching 


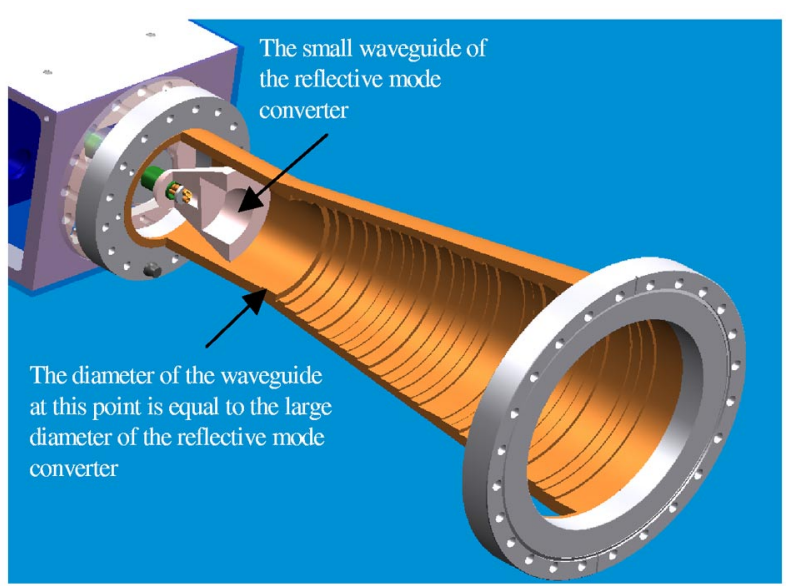

FIG. 4. (Color) End taper and mode converter. The movable cup at the end of the taper reflects the $\mathrm{TE}_{01}$ mode into the $\mathrm{TE}_{02}$ mode, and vice versa.

algorithms the reader is referred to Ref. [10]. In circularly symmetric structures $\mathrm{TE}_{0 n}$ modes do not couple with $\mathrm{TM}_{0 n}$ modes. This allows us to optimize the speed of our mode matching code. Because of this optimized speed we can vary the parameters of each section and optimize their performance based on a goal function. The goal function used in this code was the multiplication of all the magnitudes of the scattering parameters of interest. The goal function for the input taper is the magnitude of the coupling coefficient between the $\mathrm{TE}_{01}$ mode at the input and output of the taper times the magnitude of the coupling coefficient between the incident $\mathrm{TE}_{02}$ mode at the output port of the taper and the reflected $\mathrm{TE}_{02}$ mode at the same port. The resultant shape of the input taper and its computed frequency response are shown in Figs. 5 and 6 . The resultant shape of the end taper and its computed frequency response are shown in Figs. 7 and 8.

\section{Pulse compression system response}

All measurements were performed using an HP8510C network analyzer with the results examined in the time

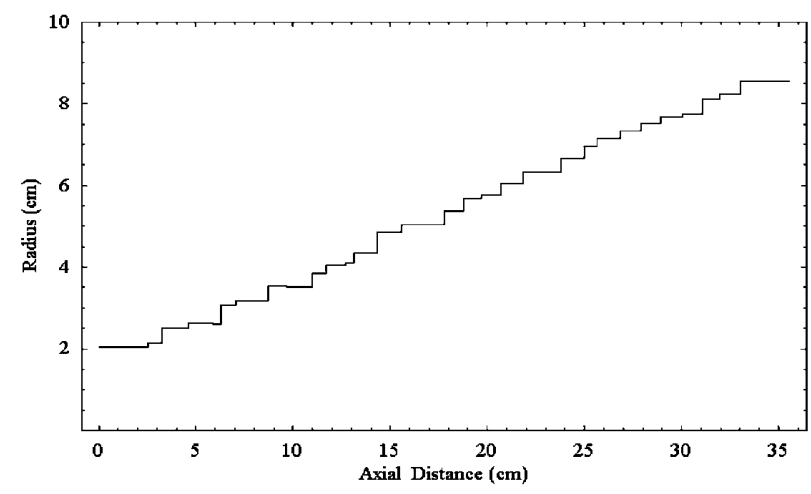

FIG. 5. Input taper designs produced by the mode matching optimization code.

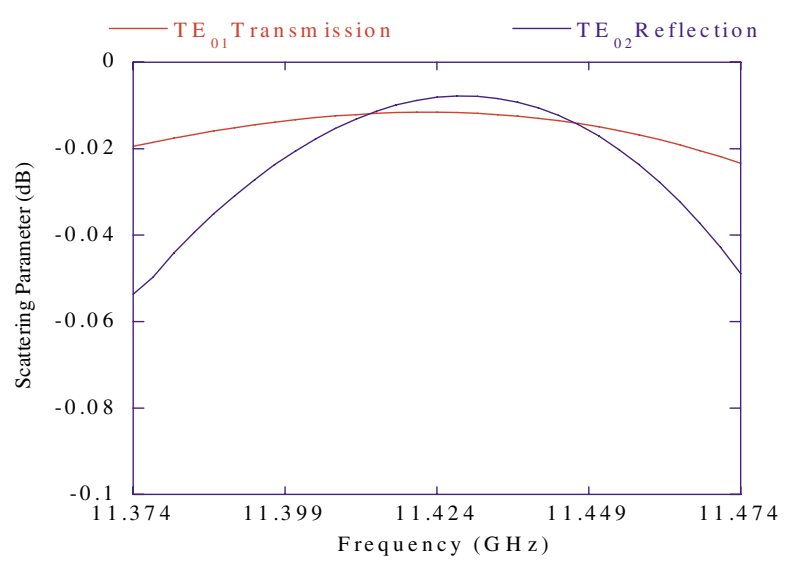

FIG. 6. (Color) The simulated frequency response of the input taper.

domain using a PC. The system is shown in Fig. 9. The

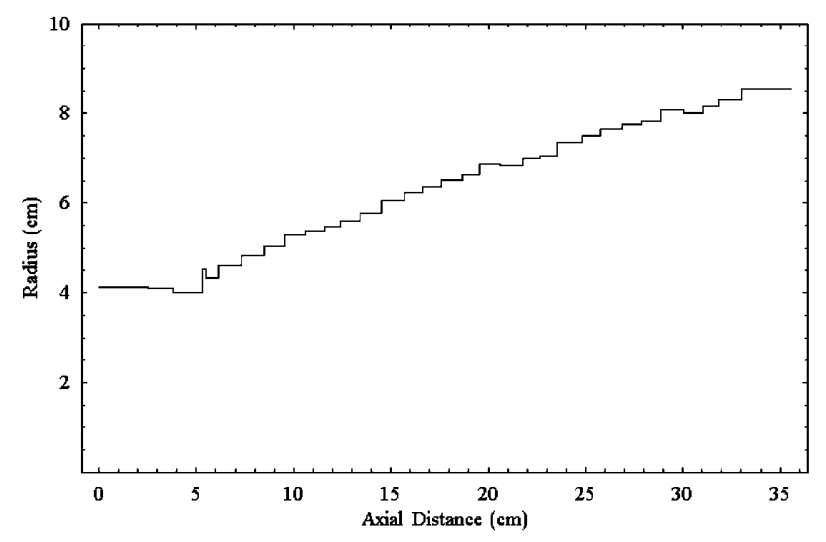

FIG. 7. End taper designs produced by the mode matching optimization code.

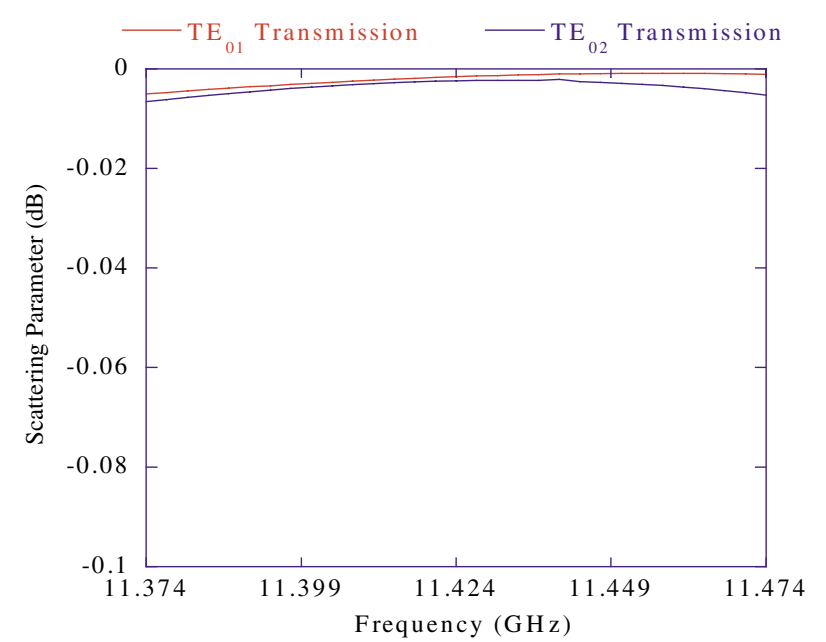

FIG. 8. (Color) The simulated frequency response of the end taper. 


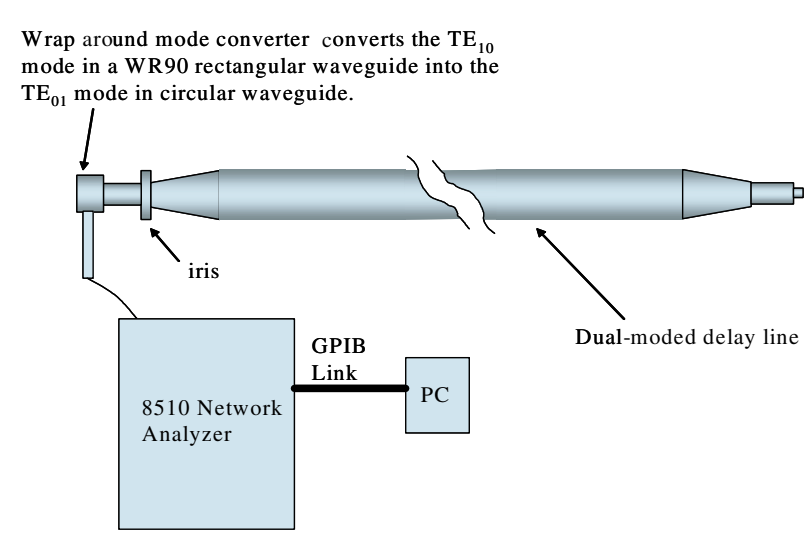

FIG. 9. (Color) Experimental setup for characterizing the resonant delay line pulse compression system.

frequency domain measurements, shown in Fig. 10, were transferred to the PC via a General Purpose IO Bus link and multiplied by the fast-Fourier transform (FFT) of a maximally flat pulse modulating an $11.424 \mathrm{GHz}$ signal. If we define the compressed pulse width as $\tau$ (equal to 2 times the round-trip time of the $\mathrm{rf}$ through the delay line), the input pulse width should be of the form $n \tau$, where $n$ is an integer equal to the compression ratio. For a given compression ratio, the phase of the input pulse should be reversed $180^{\circ}$ at the time $\tau(n-1)$. In our case the value of $\tau$ is chosen to be $400 \mathrm{~ns}$. The test pulse has the following form:

$$
V_{\text {in }}(t)=\frac{1}{\sqrt{1+\left(\frac{2 t}{\tau n}\right)^{2 l}}}-\frac{2}{\sqrt{1+\left(2 \frac{\left(t-\frac{\tau(n-1)}{2}\right)}{\tau}\right)^{2 k}}},
$$

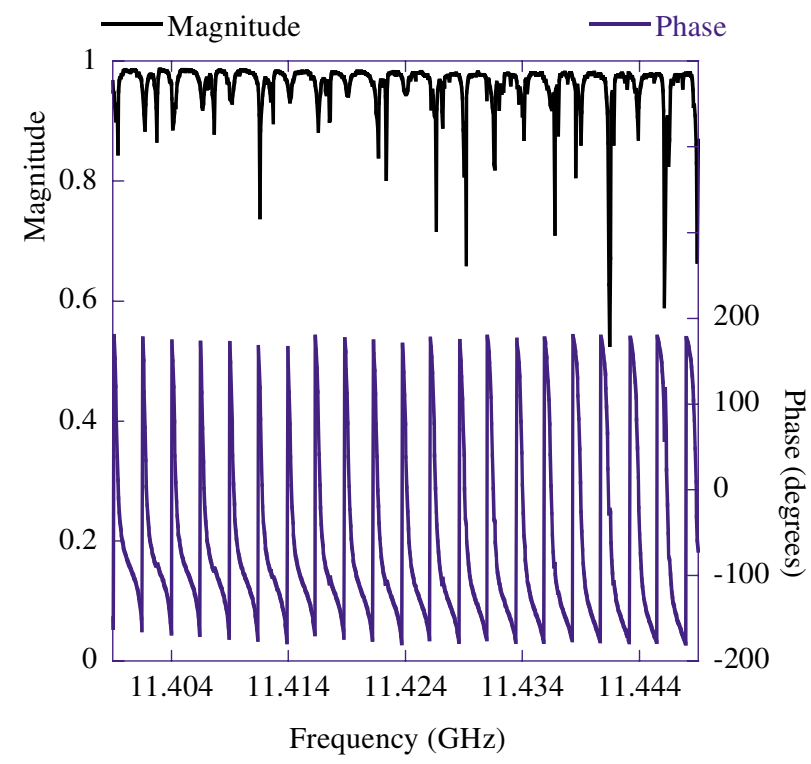

FIG. 10. (Color) Frequency response of the dual-moded delay line with an iris inserted between the mode launcher and the delay line. The iris has a reflection coefficient of 0.607 .

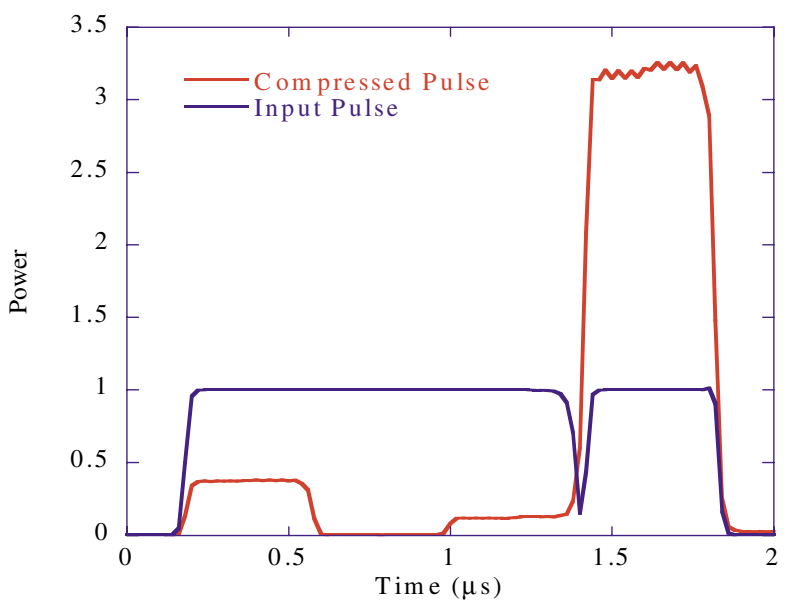

FIG. 11. (Color) Pulse compression system response.

where $l$ controls the pulse rise time and $k$ controls the phase reversal rise time. The time domain output is produced by taking the inverse FFT of this frequency domain product. Note that once we obtain the frequency characteristics of the system from the network analyzer, we can calculate the time domain response for any arbitrary input pulse.

The results of these measurements are shown in Fig. 11. Taking into account the computed response of the tapers, the theoretical losses of the delay line, and the losses due to external mode launchers, the system pulse compression gain of 3.2 is very close to the theoretical estimates [3]. Note that the width of the time bins is governed only by the delay though the line. The $400 \mathrm{~ns}$ phase flip happens only at the last time bin. The first three time bins are free to take any value. Their width is $400 \mathrm{~ns}$ indicating two round-trips, one for the $\mathrm{TE}_{01}$ mode and another for the $\mathrm{TE}_{02}$ mode.

\section{SUMMARY}

We have demonstrated a new idea for multimoded reflective delay lines. We designed and built the rf components needed for a dual-moded system. Our experimental results agreed well with theory. We also presented designs for components to build a pulse compression system that can operate with this delay lines. The idea of multiple modes need not stop at two modes. The difficulty of designing multiple function tapers can be alleviated with the use of modern optimization tools and fast simulation codes.

\section{ACKNOWLEDGMENTS}

The Author wishes to thank C. Nantista for his insight and help in the design of the end mode converters. We thank R. Ruth and P. Wilson for many useful discussions. We also thank Jose Chan for his dedicated effort to bring the experimental setup together. 
[1] "International Study Group Progress Report On Linear Collide Development," by International Study Group and High-Energy Accelerator Organization, SLAC Report No. SLAC-R-559, No. SLAC-R-0559, No. SLAC-559, No. SLAC-0559, KEK REPORT No. 2000-7, Report No. LCC-0042, 2000, ISG Progress Report, 2000.

[2] S. G. Tantawi, C. Nantista, N. Kroll, Z. Li, R. Miller, R. Ruth, P. Wilson, and J. Neilson, Phys. Rev. ST Accel. Beams 5, 032001 (2002).

[3] S. G. Tantawi, R. J. Loewen, C. D. Nantista, and A. E. Vlieks, IEEE Trans. Microwave Theory Tech. 47, 2539 (1999).

[4] P. B. Wilson, Z. D. Farkas, and R. D. Ruth, in Proceedings of the Linear Accelerator Conference, Albuquerque, NM, 1990 (LANL, Albuquerque, NM,
1990); also in SLAC Report No. SLAC-PUB-5330, 1990.

[5] Sami G. Tantawi, in Proceedings of the 9th International Workshop on Linear Colliders, 2002, http://wwwconf.slac.stanford.edu/lc02/wg2/session_3.htm.

[6] Sergey Kazakov, in Proceedings of the KEK-SLAC ISG9 Meeting, KEK, Japan, 2002, http://lcdev.kek.jp/ISG/ ISG9.html

[7] High Frequency Structure Simulator (HFSS), Ansoft Corporation, www.ansoft.com

[8] H. Flugel and E. Kuhn, IEEE Trans. Microwave Theory Tech. 36, 332 (1988).

[9] The splitting of the end mode converter from the taper was suggested by C. Nantista (private communication).

[10] Jeff M. Neilson et al., IEEE Trans. Microwave Theory Tech. 37, 1165 (1989). 